\title{
Performance analysis of multiple-input multiple- output singular value decomposition transceivers during fading and other cell interference
}

\author{
P.J. Smith, L.M. Garth and M. Shafi
}

\begin{abstract}
A generalised method is derived to compute the error probabilities of singular value decomposition (SVD)-based receivers for a multiple-input multiple-output (MIMO) system with uncoded transmission. The method can be used for a wide class of flat fading environments, including independent and identically distributed (i.i.d.) and semi-correlated Rayleigh and i.i.d. Ricean channels. Although the method is applied to equal-power binary phase shift keying, it can easily be extended to higher-order M-ary phase shift keying (M-PSK) and M-ary quadrature amplitude modulation (M-QAM) signal constellations and adaptive 'water-filling' schemes. The error probability curves derived from closed-form formulas and simulations demonstrate very close agreement. The error performances of channel inversion, minimum mean square error and zero forcing receivers are compared with the SVD receiver for a single-user system. The impact of multiple users is considered by studying the performance of an adaptive MIMO SVD transmission scheme operating in a cellular environment. In particular, the effect of inter-cell interference on the performance of the scheme is quantified, modelling the interference as increased Gaussian noise. A number of cellular layouts are examined and the impact of the resulting singal-to-interference and noise ratio on the constellation sizes that can be supported, the BER and so on is considered. The primary metric used for our performance analysis is the error-free transmission rate, which is derived for our adaptive system. For the cellular scenarios considered, it can be found that the effect of interference is considerable and the performance of the adaptive MIMO SVD scheme is only marginally better than that provided by conventional diversity methods.
\end{abstract}

\section{Introduction}

The pioneering work of Telatar [1] and Foschini and Gans [2] has resulted in immense interest in multiple-input multiple-output (MIMO) systems. They offer the promise of large system capacities, and thus are being considered for fourth generation wireless systems. However, the majority of work in this area has focussed on single-user MIMO systems, and recent results [3] suggest that the promised rates may not be available in cellular systems. Hence, in this paper we study the performance of both fixed and adaptive MIMO singular value decomposition (SVD) transmission schemes in a cellular environment and make the following contributions:

- We present a generalised method that can be used to derive the exact symbol-error probability of fixed SVD-based MIMO receivers using uncoded transmission. We demonstrate the method for independent and identically distributed (i.i.d.) and semi-correlated Rayleigh and i.i.d. Ricean channels. Our results provide new insights in

(C) The Institution of Engineering and Technology 2008

doi:10.1049/iet-map:20060262

Paper first received 27th September 2006 and in revised form 21st October 2007 P.J. Smith and L.M. Garth are with the Department of Electrical and Computer Engineering, University of Canterbury, Private Bag 4800, Christchurch, New Zealand

M. Shafi is with Telecom New Zealand Limited, Wellington, New Zealand

E-mail: p.smith@elec.canterbury.ac.nz understanding the error performance of MIMO systems. For example, when the number of antennas is increased from two to four at both the transmit and receive ends, although the ergodic capacity increases, the error performance degrades. Hence, we are able to quantify the tradeoff between ergodic capacity and system outage.

- We compare the fixed SVD receiver error performance with channel inversion (CI), minimum mean square error (MMSE) and zero forcing (ZF) receivers and show that for all types of channels and signal to noise ratio (SNR), the MMSE receiver outperforms the other receivers.

- For the cellular environment, we show that the performance of an adaptive MIMO SVD system is heavily degraded under low signal-to-interference and noise ratio (SINR) conditions. As a result, the error free rate, averaged over the cellular interference, is only marginally better than that provided by conventional diversity methods.

- We compare the performance of the adaptive scheme with that of a fixed modulation system employing MIMO-MMSE receivers and demonstrate the improvements offered by the adaptive SVD approach.

Finally, as we have done in the point-to-point case, our analytical method for the Rayleigh channel in the cellular environment can be extended to derive results for semicorrelated Rayleigh and i.i.d. Ricean channels, but this is beyond the scope of the paper.

A number of researchers have analytically evaluated the performance of single-user MIMO systems under different channel conditions and receiving architectures [4-6]. 
However, the subject of MIMO performance under other cell interference has not had the same degree of robust discussion.

The layout of the paper is as follows. In Section 2, we define the fixed MIMO SVD transmission scheme and describe the cellular layouts considered. In Section 3, we derive the expressions for the exact BPSK symbol error rate probabilities for SVD-based transmission. In Section 5 we extend the fixed BPSK system to an adaptive modulation scheme and derive its error free rate. We give our simulation results in Section 6 and conclude the paper in Section 7.

\section{Background}

Here, we consider a single-user MIMO system with perfect channel state information (CSI) at both the transmitter and receiver, operating both in point-to-point and cellular environments. For the cellular environment, the effect of the interference on the MIMO system is catered for by a simple scaling of the additive noise power. Hence, we assume that the interference is Gaussian and unknown at the receiver.

\subsection{Channel model and SVD transmission}

We model the channel using a variety of flat fading models including uncorrelated and correlated Rayleigh models and a Ricean model. For a MIMO system with $n_{\mathrm{T}}$ transmit and $n_{\mathrm{R}}$ receive antennas, the received signal can be written

$$
\boldsymbol{r}=\boldsymbol{H} \boldsymbol{s}+\boldsymbol{n}
$$

where $\boldsymbol{r}$ is the $n_{\mathrm{R}} \times 1$ received signal vector, $\boldsymbol{s}$ the complex $n_{\mathrm{T}} \times 1$ transmitted signal vector and $\boldsymbol{H}$ is an $n_{\mathrm{R}} \times n_{\mathrm{T}}$ complex channel gain matrix. The additive white gaussian noise (AWGN) vector $\boldsymbol{n}$ consists of $n_{\mathrm{R}}$ independent noise plus interference components with $\operatorname{var}\left(\operatorname{Re}\left[n_{i}\right]\right)=\operatorname{var}\left(\operatorname{Im}\left[n_{i}\right]\right)=\sigma^{2} / 2$.

If we have perfect CSI, we can perform a SVD of $\boldsymbol{H}=\boldsymbol{U D} \boldsymbol{V}$, where $\boldsymbol{U}$ and $\boldsymbol{V}$ are unitary matrices and $\boldsymbol{D}$ a diagonal matrix with entries $\sqrt{\lambda_{k}}, k=1, \ldots, m$. Here, we denote length $m=\min \left(n_{\mathrm{T}}, n_{\mathrm{R}}\right), n=\max \left(n_{\mathrm{T}}, n_{\mathrm{R}}\right)$ and the $\lambda_{k}$ 's are the distinct eigenvalues of

$$
\boldsymbol{W}=\left\{\begin{array}{lll}
\boldsymbol{H} \boldsymbol{H}^{\dagger} & \text { for } & n_{\mathrm{R}} \leq n_{\mathrm{T}} \\
\boldsymbol{H}^{\dagger} \boldsymbol{H} & \text { for } & n_{\mathrm{T}}<n_{\mathrm{R}}
\end{array}\right.
$$

where $(\cdot)^{\dagger}$ denotes the conjugate transpose.

As per conventional SVD-based spatial multiplexing methods $[7,8]$, we precode our $m$-dimensional BPSK symbol vector $\boldsymbol{b}$ by multiplying it by $\boldsymbol{V}^{\dagger}$ and decode our received observation vector $\boldsymbol{r}$ by multiplying it by $\boldsymbol{U}^{\dagger}$. Defining $\boldsymbol{s}=\boldsymbol{V}^{\dagger} \boldsymbol{b}, \boldsymbol{y}=\boldsymbol{U}^{\dagger} \boldsymbol{r}$, and $\tilde{\boldsymbol{n}}=\boldsymbol{U}^{\dagger} \boldsymbol{n}$ and transforming (1) by using the orthonormality of $\boldsymbol{U}$ and $\boldsymbol{V}$, the decoder output has the form

$$
\boldsymbol{y}=\boldsymbol{D b}+\tilde{\boldsymbol{n}}
$$

Due to the orthonormality of $\boldsymbol{U}^{\dagger}$, the transformed noise vector $\tilde{\boldsymbol{n}}$ remains white Gaussian with $\operatorname{var}\left(\operatorname{Re}\left[\tilde{n}_{i}\right]\right)=\operatorname{var}\left(\operatorname{Im}\left[\tilde{n}_{i}\right]\right)=\sigma^{2} / 2$. Because $\boldsymbol{D}$ is diagonal, the MIMO channel (1) has been transformed into $m$ parallel channels of the form

$$
y_{k}=\sqrt{\lambda_{k}} b_{k}+\tilde{n}_{k} \quad k=1, \ldots, m
$$

Power allocation algorithms could be used on the parallel channels by sending different sized signal constellations down each channel. However, in this paper, we only consider the equal power distribution method.

\section{9-Cell Cluster:}

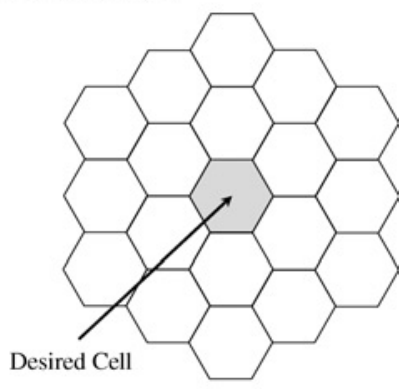

Cell Sectorisation Options:

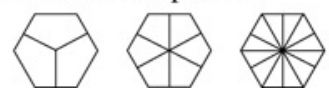

Fig. 1 Cluster of 19 cells and a cell split into 3, 6 and 12 sectors

\subsection{Cellular layouts}

For the cellular environment, to study the impact of intercell interference, we consider a system based on an industry standard [9] 19-cell cluster of hexagonal cells as shown in Fig. 1. Each of the cells employs either 3, 6 or 12 sectors as shown in the same figure. Furthermore, among neighbouring cells one may either use the same frequency $F=1$, or employ a reuse pattern of $3, F=1 / 3$, as discussed in [3]. The user is connected to the base station with the strongest signal. All other base stations are then deemed as interferers. By randomly placing users in the desired and surrounding cells, and by assuming appropriate values for the path-loss exponent and log-normal shadowing, one can find an SINR, denoted $\Gamma$. The SINR can now be generated by the procedure in [3]. The resulting SINR cumulative distributions are shown in Fig. 2. The different scenarios are referred to as R1S3, R1S6, R1S12, R3S3, R3S6 and R3S12 (the notation R1S3 stands for a reuse of 1 with 3 sectors).

Returning to the SVD-transformed channel model (4), note that for the cellular environment $\sigma^{2}$ represents both the additive noise and the interference from the surrounding cells. Hence, interference is modelled as increased Gaussian noise and $\sigma^{2}$, the interference-plus-noise power, is dependent on the cellular layout. The values of SINR $\Gamma$ are related to $\sigma^{2}$ through the formula $\sigma^{2}=n_{\mathrm{T}} / \Gamma$. With equal power allocation to the antennas, the SINR per eigenmode is $\Gamma / n_{\mathrm{T}}$. The corresponding instantaneous SINR is defined by $\alpha_{k}=\lambda_{k} \Gamma / n_{\mathrm{T}}$ for $k=1, \ldots, n_{\mathrm{T}}$. In the adaptive scheme discussed in Section 5, the symbols $b_{k}$ can be selected adaptively from various constellations according to the instantaneous SINR value $\alpha_{k}$.

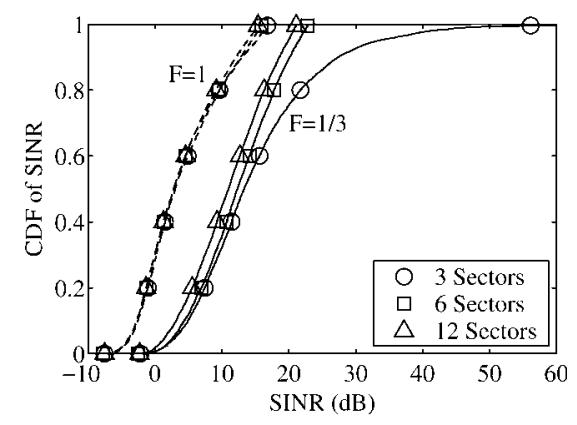

Fig. 2 SINR CDF curves for all six cellular scenarios 


\section{$3 \quad$ Exact BPSK symbol error probabilities}

In this section, we consider the most basic case of transmitting symbols from identical BPSK constellations down each eigenchannel. Normalising the transmitted BPSK symbols by $n_{\mathrm{T}}$ to keep the total transmitted signal power constant, we let $\operatorname{Prob}\left(b_{k}=1 / \sqrt{n_{\mathrm{T}}}\right)=\operatorname{Prob}\left(b_{k}=-1 / \sqrt{n_{\mathrm{T}}}\right)=1 / 2$. The optimal detector for each parallel channel is then

$$
\widehat{b}_{k}=\operatorname{sgn}\left(\operatorname{Re}\left[y_{k}\right]\right)
$$

Since the probability of error is the same for all combinations of positive and negative binary symbols in $\boldsymbol{b}$, the probability of an MIMO symbol error for this system is

$$
\begin{aligned}
P_{\mathrm{s}} & =1-\operatorname{Prob}\left(\widehat{b}_{1}, \widehat{b}_{2}, \ldots, \widehat{b}_{m} \text { all correct }\right) \\
& =1-\operatorname{Prob}\left(\sqrt{\frac{\lambda_{1}}{n_{\mathrm{T}}}}+\operatorname{Re}\left[\tilde{n}_{1}\right]>0, \ldots, \sqrt{\frac{\lambda_{m}}{n_{\mathrm{T}}}}+\operatorname{Re}\left[\tilde{n}_{m}\right]>0\right) \\
& =1-E\left\{\Phi\left(\frac{\sqrt{\lambda_{1}}}{\sigma}\right) \times \Phi\left(\frac{\sqrt{\lambda_{2}}}{\sigma}\right) \times \cdots \times \Phi\left(\frac{\sqrt{\lambda_{m}}}{\sigma}\right)\right\} \\
& =1-\int_{0}^{\infty} \ldots \int_{0}^{\infty} \prod_{i=1}^{m} \Phi\left(\frac{\sqrt{\lambda_{i}}}{\sigma}\right) f\left(\lambda_{1}, \ldots, \lambda_{m}\right) \mathrm{d} \lambda_{1}, \ldots, \mathrm{d} \lambda_{m} \\
& =1-\int_{\lambda}^{m} \prod_{i=1}^{m} \Phi\left(\frac{\sqrt{\lambda_{i}}}{\sigma}\right) f(\boldsymbol{\lambda}) \mathrm{d} \boldsymbol{\lambda}
\end{aligned}
$$

where $\sigma^{2}=n_{\mathrm{T}} \sigma_{n}^{2} / 2$ and $\Phi(x)$ is the cumulative distribution function of a standard Gaussian variable, that is, $\Phi(x)=\operatorname{Prob}(Z \leq x)$ for $Z \sim N(0,1)$.

The joint density of the 'unordered' eigenvalues for an i.i.d. Rayleigh fading channel is well known $[1,10]$ and with the constant $\Delta$ defined by $\Delta=\left\{\prod_{k=1}^{m}[(n-k) !(m-k) !]\right\}^{-1}$ is given by

$$
\begin{aligned}
& f_{\mathrm{I}}(\boldsymbol{\lambda})=\frac{\Delta}{m !} \exp \left\{-\sum_{k=1}^{m} \lambda_{k}\right\} \prod_{k=1}^{m} \lambda_{k}^{n-m} \prod_{i<j}^{m}\left(\lambda_{i}-\lambda_{j}\right)^{2} \\
& =\frac{\Delta}{m !} \prod_{k=1}^{m}\left(\lambda_{k}^{n-m} \mathrm{e}^{-\lambda_{k}}\right)\left|\begin{array}{ccc}
1 & \cdots & 1 \\
\lambda_{1} & \cdots & \lambda_{m} \\
\vdots & & \vdots \\
\lambda_{1}^{m-1} & \cdots & \lambda_{m}^{m-1}
\end{array}\right|^{2} \\
& =\frac{\Delta}{m !}\left|\begin{array}{ccc}
1 & \cdots & 1 \\
\lambda_{1} & \cdots & \lambda_{m} \\
\vdots & & \vdots \\
\lambda_{1}^{m-1} & \cdots & \lambda_{m}^{m-1}
\end{array}\right| \\
& \times\left|\begin{array}{ccc}
\lambda_{1}^{n-m} \mathrm{e}^{-\lambda_{1}} & \cdots & \lambda_{m}^{n-m} \mathrm{e}^{-\lambda_{m}} \\
\lambda_{1}^{n-m+1} \mathrm{e}^{-\lambda_{1}} & \cdots & \lambda_{m}^{n-m+1} \mathrm{e}^{-\lambda_{m}} \\
\vdots & & \vdots \\
\lambda_{1}^{n-1} \mathrm{e}^{-\lambda_{1}} & \cdots & \lambda_{m}^{n-1} \mathrm{e}^{-\lambda_{m}}
\end{array}\right| \\
& \triangleq \frac{C}{m !} \Upsilon(\boldsymbol{\lambda})\left|\Psi_{i j}\left(\lambda_{j}\right)\right|
\end{aligned}
$$

The last representation for this density is purposely generic. With suitable definitions of $C$ and $\Psi_{i j}$, we are able to cast each of the channel models we consider in this general form.
As shown in [1], an alternative form of the Vandermonde determinant $\boldsymbol{Y}(\boldsymbol{\lambda})$ is

$$
\begin{aligned}
\Upsilon(\boldsymbol{\lambda}) & =\left|\begin{array}{ccc}
1 & \cdots & 1 \\
\vdots & & \vdots \\
\lambda_{1}^{m-1} & \cdots & \lambda_{m}^{m-1}
\end{array}\right| \\
& =\sum_{\alpha}(-1)^{\operatorname{per}(\alpha)} \prod_{k=1}^{m} \lambda_{k}^{\alpha_{k}-1}
\end{aligned}
$$

where $\alpha$ is a permutation of $(1, \ldots, m)$. The summation runs over all possible permutations of $(1, \ldots, m)$, and $(-1)^{\operatorname{per}(\alpha)}$ gives the sign of the permutation (i.e. $\operatorname{per}(\alpha)$ represents the number of column swaps required to order $\left.\left(\alpha_{1}, \ldots, \alpha_{m}\right)\right)$. The term $\left|\Psi_{i j}\left(\lambda_{j}\right)\right|$ in (7) denotes the determinant of the $m \times m$ matrix with $i j$ th element $\Psi_{i j}\left(\lambda_{j}\right)$.

Substituting (7) and (8) into (6) gives

$$
\begin{aligned}
P_{\mathrm{s}}= & 1-\frac{C}{m !} \sum_{\alpha}(-1)^{\mathrm{per}(\alpha)} \\
& \times \int_{\lambda} \prod_{j=1}^{m}\left\{\left(\frac{\sqrt{\lambda_{j}}}{\sigma}\right) \lambda_{j}^{\alpha_{j}-1}\right\}\left|\Psi_{i j}\left(\lambda_{j}\right)\right| \mathrm{d} \boldsymbol{\lambda} \\
= & 1-\frac{C}{m !} \sum_{\alpha}(-1)^{\operatorname{per}(\alpha)} \int_{\boldsymbol{\lambda}}\left|\Phi\left(\frac{\sqrt{\lambda_{j}}}{\sigma}\right) \lambda_{j}^{\alpha_{j}-1} \Psi_{i j}\left(\lambda_{j}\right)\right| \mathrm{d} \boldsymbol{\lambda} \\
= & 1-\frac{C}{m !} \sum_{\alpha}(-1)^{\operatorname{per}(\alpha)}\left|\left[\boldsymbol{g}\left(1, \alpha_{1}\right) \cdots \boldsymbol{g}\left(m, \alpha_{m}\right)\right]\right|
\end{aligned}
$$

Here, each column vector has the form $\boldsymbol{g}\left(j, \alpha_{j}\right)=\left[g_{1}\left(j, \alpha_{j}\right), \ldots, g_{m}\left(j, \alpha_{j}\right)\right]^{\mathrm{T}}$, where

$$
g_{i}\left(j, \alpha_{j}\right)=\int_{0}^{\infty} \Phi\left(\sqrt{\lambda_{j}} / \sigma\right) \lambda_{j}^{\alpha_{j}-1} \Psi_{i j}\left(\lambda_{j}\right) \mathrm{d} \lambda_{j}
$$

Re-ordering the columns in (9) gives

$$
P_{\mathrm{s}}=1-\frac{C}{m !} \sum_{\alpha}\left|\left[\boldsymbol{g}\left(\alpha_{1}, 1\right) \cdots \boldsymbol{g}\left(\alpha_{m}, m\right)\right]\right|
$$

since reordering the columns so, $\alpha_{1}, \ldots, \alpha_{m}$ are in order yields a $(-1)^{\text {per }(\alpha)}$ factor. Fortunately, vector $\boldsymbol{g}\left(\alpha_{j}, j\right)$ is independent of $\alpha_{j}$, since $\alpha_{j}$ simply locates the eigenvalue which is being integrated. Hence, all determinants of the sum (10) are equal. Because there are $m$ ! permutations in the sum, we finally have

$$
P_{\mathrm{s}}=1-C|[\boldsymbol{g}(1) \cdots \boldsymbol{g}(m)]|
$$

where $\boldsymbol{g}(j)=\left[g_{1}(j), \ldots, g_{m}(j)\right]^{\mathrm{T}}$ and

$$
g_{i}(j)=\int_{0}^{\infty} \Phi\left(\frac{\sqrt{\lambda}}{\sigma}\right) \lambda^{j-1} \Psi_{i j}(\lambda) \mathrm{d} \lambda
$$

This is the step that makes analysis realistic as sums over $m$ ! permutations are undesirable. Note that this form of solution is valid for any channel with joint eigenvalue density given by (7). Eigenvalue densities of this form include those for the i.i.d. and semi-correlated Rayleigh and i.i.d. Ricean channels. Note that (11) can be derived directly from (6) using results in the appendix of [11]. However, (11) is the central result required for all three channel models, so the derivation is included here for completeness.

\subsection{I.i.d. Rayleigh channel}

As shown in (7), for the i.i.d. Rayleigh fading channel, the constant $C=\Delta$ and the function $\Psi_{i j}\left(\lambda_{j}\right)$ has the specific 
form $\lambda_{j}^{n-m+i-1} \mathrm{e}^{-\lambda_{j}}$. Therefore we have

$$
\begin{aligned}
g_{i}(j) & =\int_{0}^{\infty} \Phi\left(\frac{\sqrt{\lambda}}{\sigma}\right) \lambda^{n-m+j+i-2} \mathrm{e}^{-\lambda} \mathrm{d} \lambda \\
& =\xi(n-m+j+i-2, \sigma)
\end{aligned}
$$

Using the relation $\Phi(x)=1-Q(x)$, where $Q(\cdot)$ is the Gaussian tail probability, we can rewrite (13) in the generic form

$$
\begin{aligned}
\xi(r, \sigma) & \triangleq \int_{0}^{\infty} \Phi\left(\frac{\sqrt{\lambda}}{\sigma}\right) \lambda^{r} \mathrm{e}^{-\lambda} \mathrm{d} \lambda \\
& =r !-\int_{0}^{\infty} Q\left(\frac{\sqrt{\lambda}}{\sigma}\right) \lambda^{r} \mathrm{e}^{-\lambda} \mathrm{d} \lambda
\end{aligned}
$$

But as shown in [12, p. 825], the second term on the right can be simplified as

$$
\begin{aligned}
\int_{0}^{\infty} Q\left(\frac{\sqrt{\lambda}}{\sigma}\right) \lambda^{r} \mathrm{e}^{-\lambda} \mathrm{d} \lambda= & r !\left\{\frac{1}{2}[1-\mu(\sigma)]\right\}^{r+1} \\
& \times \sum_{k=0}^{r}\left(\begin{array}{c}
r+k \\
k
\end{array}\right)\left\{\frac{1}{2}[1+\mu(\sigma)]\right\}^{k}
\end{aligned}
$$

where

$$
\mu(\sigma)=\sqrt{\frac{1}{1+2 \sigma^{2}}}
$$

(Even though the noise variance $\sigma^{2}$ appears by itself, $\mu(\sigma)$ is dimensionless because of the implicit ratio of $\sigma^{2}$ with the unit signal power.) Hence, for the i.i.d. Rayleigh channel $P_{\mathrm{s}}$ can be computed in closed form using $(11)$ in conjunction with (13-16).

\subsection{Semi-correlated Rayleigh channel}

In [13], we considered the 'semi-correlated' Rayleigh channel model, where the transceiver end that only has $m$ antennas experiences spatial correlation. For example, if $n_{\mathrm{R}} \leq n_{\mathrm{T}}$ and the receiving antennas are spatially correlated, then the columns of $\boldsymbol{H}$ are i.i.d., each with covariance matrix $\Theta_{\mathrm{c}}$. Likewise, if $n_{\mathrm{R}}>n_{\mathrm{T}}$ and the transmitting antennas are spatially correlated, then the columns of $\boldsymbol{H}^{\dagger}$ have covariance $\Theta_{c}$. As shown in [11], the distinct unordered eigenvalues $\boldsymbol{\lambda}$ then have joint density

$$
f_{\mathrm{SC}}(\boldsymbol{\lambda})=\frac{\Delta}{m !} \frac{\prod_{\ell=1}^{m}[(\ell-1) !]}{\left|\Xi_{i j}\left(\gamma_{j}\right)\right|} \Upsilon(\boldsymbol{\lambda})\left|\Psi_{i j}\left(\lambda_{j}\right)\right|
$$

where $\Xi_{i j}\left(\gamma_{j}\right)=(-1)^{i-1} \gamma_{j}^{n-i+1}, \Psi_{i j}\left(\lambda_{j}\right)=\lambda_{j}^{n-m} \mathrm{e}^{-\lambda_{j} / \gamma_{i}}$ and $\boldsymbol{\gamma}=\left[\gamma_{1} \cdots \gamma_{m}\right]^{\mathrm{T}}$ is a vector containing the eigenvalues of $\Theta_{\mathrm{c}}$. Thus, error probability $P_{\mathrm{s}}$ has the form (11) with

$$
C=\left[\prod_{k=1}^{m}[(n-k) !]\left|\Xi_{i j}\left(\gamma_{j}\right)\right|\right]^{-1}
$$

and

$$
\begin{aligned}
g_{i}(j) & =\int_{0}^{\infty} \Phi\left(\frac{\sqrt{\lambda}}{\sigma}\right) \lambda^{n-m+j-1} \mathrm{e}^{-\lambda / \gamma_{i}} \mathrm{~d} \lambda \\
& =\gamma_{i}^{n-m+j} \xi\left(n-m+j-1, \sigma / \sqrt{\gamma_{i}}\right)
\end{aligned}
$$

which can be evaluated using (14-16).

\section{I.i.d. Ricean channel}

We finally compute the probability of MIMO symbol error for an i.i.d. Ricean fading channel. As discussed in [14, 15], the Ricean channel matrix $\boldsymbol{H}$ has the form

$$
\boldsymbol{H}=a \boldsymbol{H}^{\mathrm{sp}}+b \boldsymbol{H}^{\mathrm{sc}}
$$

where the specular and scattered components of $\boldsymbol{H}$ are denoted by superscripts. Matrix $\boldsymbol{H}^{\mathrm{sp}}$ is deterministic with unit magnitude elements, and the entries of $\boldsymbol{H}^{\mathrm{sc}}$ are independent, zero mean, unit variance and complex Gaussians. The parameters $a$ and $b$ satisfy $a^{2}+b^{2}=1$ so that the SNR is not scaled by the channel. In standard models [14], the specular matrix is defined as

$$
\boldsymbol{H}^{\mathrm{sp}}=\boldsymbol{a}\left(\theta_{r}\right) \boldsymbol{a}\left(\theta_{t}\right)^{\mathrm{T}}
$$

where $\boldsymbol{a}\left(\theta_{\mathrm{r}}\right)$ and $\boldsymbol{a}\left(\theta_{\mathrm{t}}\right)$ are the specular array responses at the receiver and transmitter. If a $k$-element array is linear, the response is $\boldsymbol{a}(\theta)=\left[1 \mathrm{e}^{\mathrm{j} 2 \pi d \cos (\theta)} \cdots \mathrm{e}^{\mathrm{j} 2 \pi d(k-1) \cos (\theta)}\right]^{\mathrm{T}}$, where $\theta$ is the angle of arrival or departure of the specular component, and $d$ is the antenna spacing in wavelengths. This form gives the specular matrix $\boldsymbol{a}$ rank of one. We will use this model in our numerical results, although our analysis holds for more general forms of $\boldsymbol{H}^{\mathrm{sp}}$. The strength of the line of sight (LOS) component is measured using the $K$-factor, $K=10 \log _{10}\left(a^{2} / b^{2}\right) \mathrm{dB}$

To simplify the notation, we let $\left(w_{1}, w_{2}, \ldots, w_{m}\right)$ be the eigenvalues of $\tilde{\boldsymbol{W}}=\boldsymbol{W} / b^{2}$. Thus, we have $w_{k}=\lambda_{k} / b^{2}$. We also need to define the associated version of $\boldsymbol{W}$ in (2) as the matrix $\boldsymbol{M}$, where

$$
\boldsymbol{M}= \begin{cases}\frac{a^{2}}{b^{2}} \boldsymbol{H}^{\mathrm{sp}} \boldsymbol{H}^{\mathrm{sp} \dagger} & \text { for } n_{\mathrm{R}} \leq n_{\mathrm{T}} \\ \frac{a^{2}}{b^{2}} \boldsymbol{H}^{\mathrm{sp} \dagger} \boldsymbol{H}^{\mathrm{sp}} & \text { for } n_{\mathrm{T}}<n_{\mathrm{R}}\end{cases}
$$

The eigenvalues of $\boldsymbol{M}$ are denoted by $\left(f_{1}, f_{2}, \ldots, f_{m}\right)$. In the rank one standard model for $\boldsymbol{M}$, these eigenvalues are given by: $f_{1}=\left(a^{2} / b^{2}\right) m n$ and $f_{2}=f_{3}=\cdots=f_{m}=0$.

As shown in [16], the distinct unordered eigenvalues $\boldsymbol{w}$ (with corresponding distinct eigenvalues $\boldsymbol{f}$ ) have joint density

$$
f_{\mathrm{R}}(\boldsymbol{w})=\frac{1}{m !\left|\Xi_{i j}\left(f_{j}\right)\right|} \Upsilon(\boldsymbol{w})\left|\Psi_{i j}\left(w_{j}\right)\right|
$$

where $\Psi_{i j}\left(w_{j}\right)=w_{j}^{(n-m) / 2} \mathrm{e}^{-\left(f_{i}+w_{j}\right)} I_{n-m}\left(2 \sqrt{f_{i} w_{j}}\right), \Xi_{i j}\left(f_{j}\right)=$ $f_{j}^{(n-m) / 2+i-1}$ and $I_{\ell}(\cdot)$ is a modified Bessel function. Thus, the error probability $P_{\mathrm{s}}$ has the form (11) with $C=\left|\Xi_{i j}\left(f_{j}\right)\right|^{-1}$ and

$$
\begin{aligned}
g_{i}(j)= & \int_{0}^{\infty} \Phi\left(\frac{\sqrt{w}}{\sigma / b}\right) w^{(n-m) / 2+j-1} \mathrm{e}^{-\left(f_{i}+w\right)} \\
& \times I_{n-m}\left(2 \sqrt{f_{i} w}\right) \mathrm{d} w
\end{aligned}
$$

We are unaware of an analytical solution to this integral. Nevertheless, numerical integration can be performed to evaluate $P_{\mathrm{s}}$ for the Ricean channel case.

For the special case of a rank one specular matrix, the numerator and denominator of (22) are zero for $m>2$. To cope with this situation, we need to compute the following limit

$$
\lim _{f_{2}, f_{3}, \ldots, f_{m} \rightarrow 0}\left|I_{n-m}\left(2 \sqrt{f_{i} w_{j}}\right)\right| /\left|f_{j}^{(n-m) / 2+i-1}\right|
$$


In the appendix of [4], we have derived this limit and hence can compute the joint density of the unordered eigenvalues as

$$
\begin{aligned}
f_{\mathrm{R} 1}(\boldsymbol{w})= & \frac{(-1)^{m-1} f_{1}^{-(n+m) / 2+1} \mathrm{e}^{-f_{1}}}{m ! \prod_{k=2}^{m}[(n-k) !(m-k) !]} \\
& \times \sum_{\alpha}(-1)^{\operatorname{per}(\alpha)}\left|\boldsymbol{r}_{\alpha_{1}} \boldsymbol{r}_{\alpha_{2}} \cdots \boldsymbol{r}_{\alpha_{m}}\right|
\end{aligned}
$$

In (25), the vectors $\boldsymbol{r}_{\alpha_{1}}, \boldsymbol{r}_{\alpha_{2}}, \ldots, \boldsymbol{r}_{\alpha_{m}}$ are defined by

$$
\begin{aligned}
\boldsymbol{r}_{\alpha_{j}}= & w_{j}^{n-m+\alpha_{j}-1} \mathrm{e}^{-w_{j}} \\
& \times\left[w_{j}^{-(n-m) / 2} I_{n-m}\left(2 \sqrt{f_{1} w_{j}}\right) 1 w_{j} \cdots w_{j}^{m-2}\right]^{\mathrm{T}}
\end{aligned}
$$

Following the same methodology as in (9)-(11), we are able to remove the summation of permutations in (25). For the rank one case, this leads to a $P_{\mathrm{s}}$ of the form (11) with

$$
C=\frac{(-1)^{m-1} f_{1}^{-(n+m) / 2+1}}{\prod_{k=2}^{m}[(n-k) !(m-k) !]}
$$

The first row of vector $\boldsymbol{g}(j), g_{1}(j)$, is of the form shown in (23), and the other rows $(2 \leq i \leq m)$ have elements of the form

$$
\begin{aligned}
g_{i}(j) & =\int_{0}^{\infty} \Phi\left(\frac{\sqrt{w}}{\sigma / b}\right) w^{n-m+j+i-3} \mathrm{e}^{-w} \mathrm{~d} w \\
& =\xi(n-m+j+i-3, \sigma / b)
\end{aligned}
$$

\section{Adaptive modulation and error-free rate}

We now consider extending our fixed SVD system to an adaptive modulation system by adaptively selecting between BPSK, 4-QAM and 16-QAM symbol constellations. In each case, the average symbol power is unity. Hence, for BPSK, $b_{k} \in\{-1,+1\}$, for 4-QAM, $b_{k} \in\{ \pm 1 \pm j\} / \sqrt{2}$ and for 16-QAM, $b_{k} \in\{(2 r-3)+$ $j(2 s-3)\} / \sqrt{10}$, for $r, s \in\{0,1,2,3\}$.

These constellations are selected on the basis of an eigenvalue threshold vector $\boldsymbol{q}=\left(q_{1}, q_{2}, q_{3}\right)$, where the entries of $\boldsymbol{q}$ depend on $n_{\mathrm{T}}$ and $\Gamma$ and the number of elements of $\boldsymbol{q}$ is equal to the number of modulation schemes in the set. The exact dependence is not shown for ease of notation. When $\lambda_{k}<q_{1}$, the eigenmode is considered too weak for communication and transmission is suspended. When $q_{1} \leq \lambda_{k}<q_{2}$, BPSK is used. When $q_{2} \leq \lambda_{k}<q_{3}$, 4-QAM is used and when $q_{3} \leq \lambda_{k}, 16$-QAM is used. The threshold vector, $\boldsymbol{q}$, is selected on the basis of a target bit error rates (BER), denoted by $\mathrm{BER}_{\mathrm{t}}$. Hence, for a particular SINR value and a target of $\mathrm{BER}_{\mathrm{t}}$, the instantaneous BER of BPSK is BER $_{\mathrm{t}}$ when $\lambda_{k}=q_{1}$, the instantaneous BER of 4-QAM is BER then $_{k}=q_{2}$ and the instantaneous BER of 16-QAM is BER $\mathrm{B}_{\mathrm{t}}$ when $\lambda_{k}=q_{3}$. The target BER is therefore the maximum BER that the system experiences.

\subsection{Bit error rates}

For a particular value of the SINR and $\lambda_{k}$, consider the received signal $y_{k}$ in (4). The BER values for the three different modulations can be approximated from the standard result, $\mathrm{BER} \gtrsim P($ symbol error $) / \log _{2} M$ where $M$ is the size of the constellation [12]. This gives the BER results [12]
$P_{e, 1}(\lambda, \Gamma)=1-Q\left(-\sqrt{2 \alpha_{k}}\right)$ for BPSK

$$
\begin{aligned}
P_{e, 2}(\lambda, \Gamma)= & \left(1-Q^{2}\left(-\sqrt{\alpha_{k}}\right)\right) / 2 \text { for 4-QAM } \\
P_{e, 3}(\lambda, \Gamma)= & \frac{1}{4}-\frac{1}{16} Q(-\beta)-\frac{1}{16} Q(\beta)-\frac{3}{16} Q^{2}(-\beta) \\
& +\frac{1}{8} Q(\beta) Q(-\beta) \text { for 16-QAM }
\end{aligned}
$$

where $\alpha_{k}=\lambda_{k} \Gamma / n_{\mathrm{T}}, \beta=\sqrt{2 \alpha_{k} / 10}$ and $Q(\cdot)$ is the tail probability function for a standard Gaussian variable. Using the BER results in (28), the thresholds in $\boldsymbol{q}$ can be computed by numerical solution of, for example, $P_{e, 1}(\lambda, \Gamma)=q_{1}$.

The joint densities of the 'unordered' eigenvalues for three flat fading channels have been discussed in detail in Section 3, but for the cellular scenario we concentrate only on the i.i.d. Rayleigh fading channel with joint density (7). Integrating (7) over $\lambda_{2}, \ldots, \lambda_{n_{\mathrm{T}}}$ and defining $\lambda=\lambda_{1}$ as an arbitrary eigenvalue leads to the density of $\lambda$ as [1]

$$
\begin{aligned}
f(\lambda)= & \sum_{i=1}^{n_{\mathrm{T}}} \frac{(i-1) ! \lambda^{n_{\mathrm{R}}-n_{\mathrm{T}}}}{n_{\mathrm{T}}\left(i-1+n_{\mathrm{R}}-n_{\mathrm{T}}\right) !} \\
& \times \exp (-\lambda)\left[L_{i-1}^{\left(n_{\mathrm{R}}-n_{\mathrm{T}}\right)}(\lambda)\right]^{2}
\end{aligned}
$$

where $L_{k}^{(n)}(\cdot)$ is a generalised Laguerre polynomial. This density is required in Section 5.1.1 to compute the error free rate.

5.1.1 Error free rate: In this paper, we are interested in the performance of a particular type of adaptive scheme. Hence, we do not consider the channel capacity as in [3]. In assessing the performance of the scheme, the usual BER metric can be misleading because of the need to compare systems with different or variable modulations. Hence, we consider a metric which encapsulates both the BER and constellation size and is directly comparable across different systems. This metric is the error free rate, $R_{\mathrm{T}}$, measured in bps $/ \mathrm{Hz}$, which can also be defined as the number of correctly received bits per symbol period. In the adaptive SVD scheme, $R_{\mathrm{T}}$ can approach $4 n_{\mathrm{T}}$ at high SINR, since each of the $n_{\mathrm{T}}$ antennas can employ 16-QAM. Similarly, at low SINR, $R_{\mathrm{T}}$ can approach zero when all eigenmodes suspend transmission. We focus on the mean value, $R=\mathrm{E}\left(R_{\mathrm{T}}\right)$. Hence, $R_{\mathrm{T}}$ is defined as the rate for a fixed SINR and $R$ is the overall rate, averaged over the SINR distribution.

For a single-user MIMO link in the desired cell, at a given SINR, the error free rate on the $i$ th eigenchannel is denoted $R_{i}$, and the total is $R_{\mathrm{T}}=\sum_{i=1}^{n_{\mathrm{T}}} R_{i}=n_{\mathrm{T}} \bar{R}$, where $\bar{R}$ is the average rate. The overall rate is therefore defined by

$$
R=n_{\mathrm{T}} \mathrm{E}(\bar{R})=n_{\mathrm{T}} \mathrm{E}\left(R_{o}\right)
$$

where $R_{\mathrm{o}}$ is the error free rate of an arbitrary eigenmode. We denote an arbitrary eigenvalue by $\lambda$. The eigenvalue $\lambda$ is used to select the constellation, so that the probabilities of the constellations being adopted can be related to $\lambda$ as follows

$$
\begin{aligned}
P(\mathrm{BPSK}) & =p_{1}=P\left(q_{1} \leq \lambda<q_{2}\right) \\
P(4-\mathrm{QAM}) & =p_{2}=P\left(q_{2} \leq \lambda<q_{3}\right) \\
P(16-\mathrm{QAM}) & =p_{3}=P\left(q_{3} \leq \lambda\right)
\end{aligned}
$$

The probabilities in (31) can be evaluated in closed form by direct integration of (29). This is conveniently performed in a symbolic manipulation package, such as Maple, although 
the exact results can also be written out with some extra effort. For reasons of space, these results are not presented here. Note that the values of $p_{1}, p_{2}$ and $p_{3}$ are also dependent on $n_{\mathrm{T}}$ and $\Gamma$, but again this dependence is not shown.

For each constellation, a different number of bits per symbol are used ( 1 for BPSK, 2 for 4-QAM and 4 for 16-QAM), which are placed in the vector, $\boldsymbol{B}=\left(B_{1}, B_{2}\right.$, $\left.B_{3}\right)=(1,2,4)$. With this notation in place, we can derive $R$ as follows. First we compute $\mathrm{E}\left(R_{\mathrm{T}} \mid \Gamma\right)$, the mean of $R_{\mathrm{T}}$ for a given SINR. Then, we average over the SINR to obtain $R$ as follows

$$
\begin{aligned}
\mathrm{E}\left(R_{\mathrm{T}} \mid \Gamma\right) & =n_{\mathrm{T}} \mathrm{E}\left(R_{o} \mid \Gamma\right) \\
& =n_{\mathrm{T}} \sum_{i=1}^{3} p_{i} \mathrm{E}\left(R_{o} \mid \Gamma, \text { constellation } i\right) \\
& =n_{\mathrm{T}} \sum_{i=1}^{3} p_{i} B_{i}\left[1-\mathrm{E}\left(\mathrm{BER}_{o} \mid \Gamma, \text { constellation } i\right)\right]
\end{aligned}
$$

where $\mathrm{BER}_{o}$ is the instantaneous BER of an arbitrary eigenmode. Averaging over $\mathrm{BER}_{o}$ allows (32) to be rewritten as

$$
\mathrm{E}\left(R_{\mathrm{T}} \mid \Gamma\right)=n_{\mathrm{T}} \sum_{i=1}^{3} p_{i} B_{i}\left[1-\int_{q_{i}}^{q_{i+1}} P_{e, i}(\lambda, \Gamma) f_{i}(\lambda) \mathrm{d} \lambda\right]
$$

where $f_{i}(\lambda)$ is the density of $\lambda$ conditioned on constellation $i$ being used. Hence, $f_{i}(\lambda)=f(\lambda) / p_{i}$ for $q_{i} \leq \lambda<q_{i+1}, i=1$, 2,3 and $q_{4}=\infty$. Substituting $f_{i}(\lambda)$ into (33) gives

$$
\mathrm{E}\left(R_{\mathrm{T}} \mid \Gamma\right)=n_{\mathrm{T}} \sum_{i=1}^{3} B_{i}\left[p_{i}(\Gamma)-\int_{q_{i}(\Gamma)}^{q_{i+1}(\Gamma)} P_{e, i}(\lambda, \Gamma) f(\lambda) \mathrm{d} \lambda\right]
$$

where the dependence of $p_{1}, p_{2}, p_{3}$ and $q_{1}, q_{2}, q_{3}$ on the value of $\Gamma$ is now explicitly shown. Averaging (34) over $N_{\text {SINR }}$ simulated values of the SINR gives

$$
\begin{aligned}
R= & \frac{1}{N_{\mathrm{SINR}}} \sum_{k=1}^{N_{\mathrm{SINR}}} n_{\mathrm{T}} \sum_{i=1}^{3} B_{i} \\
& \times\left[p_{i}\left(\Gamma_{k}\right)-\int_{q_{i}\left(\Gamma_{k}\right)}^{q_{i+1}\left(\Gamma_{k}\right)} P_{e, i}\left(\lambda, \Gamma_{k}\right) f(\lambda) \mathrm{d} \lambda\right]
\end{aligned}
$$

The finite range integral in (35) has to be computed numerically, although we note that if the range was $[0, \infty)$ then standard methods [17] could be used. In all our calculations, $N_{\text {SINR }}=500$ was used. Increasing $N_{\text {SINR }}$ to 5000 was also tested and results were found to be only marginally different. Note that it is also possible to analyse the BER of the adaptive system using the conditioning approach in (32) and (33). However, this is beyond the scope of the paper.

\section{Simulation results}

\subsection{Single-user case without inter-cell interference}

We first consider the fixed SVD system performance using BPSK without inter-cell interference. Fig. 3 shows our calculated and simulated symbol error probabilities against SNR for $(2,2),(2,4),(4,2)$ and $(4,4)$ MIMO systems for i.i.d channels. Our calculations line up very well with our simulations that are based on 500000 Monte Carlo runs.

We also compare our results for the SVD method with the CI method [18], which requires CSI at the transmitter, as

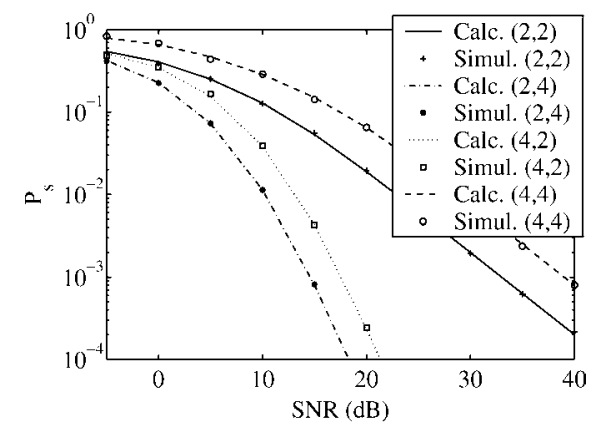

Fig. 3 SVD symbol error probabilities for i.i.d. Rayleigh case

well as the classical linear combiners using MMSE and ZF methods, which require CSI only at the receiver. The CI method works for $n_{\mathrm{T}} \geq n_{\mathrm{R}}$ and involves sending $\widetilde{\boldsymbol{s}}=\boldsymbol{H}^{\dagger}\left(\boldsymbol{H} \boldsymbol{H}^{\dagger}\right)^{-1} \boldsymbol{b}$. Unlike [18], to keep the average transmit power equal to one, we need to normalise by the rms power of $\tilde{\boldsymbol{s}}$. But, we can write

$$
\begin{aligned}
\mathrm{E}\left\{\| \boldsymbol{H}^{\dagger}\right. & \left.\left(\boldsymbol{H} \boldsymbol{H}^{\dagger}\right)^{-1} \boldsymbol{b} \|^{2}\right\} \\
& =\operatorname{Tr}\left[\boldsymbol{H}^{\dagger}\left(\boldsymbol{H} \boldsymbol{H}^{\dagger}\right)^{-1} \mathrm{E}\left\{\boldsymbol{b} \boldsymbol{b}^{\dagger}\right\}\left(\boldsymbol{H} \boldsymbol{H}^{\dagger}\right)^{-\dagger} \boldsymbol{H}\right] \\
& =\frac{1}{n_{\mathrm{T}}} \operatorname{Tr}\left[\left(\boldsymbol{H} \boldsymbol{H}^{\dagger}\right)^{-1}\right]=\frac{1}{n_{\mathrm{T}}} \sum_{k=1}^{n_{\mathrm{R}}} \frac{1}{\lambda_{k}}
\end{aligned}
$$

Therefore at the receiver we observe

$$
\boldsymbol{y}^{\mathrm{CI}}=\left(\frac{1}{n_{\mathrm{T}}} \sum_{k=1}^{n_{\mathrm{R}}} \frac{1}{\lambda_{k}}\right)^{-1 / 2} \boldsymbol{b}+\boldsymbol{n}
$$

and decode

$$
\widehat{\boldsymbol{b}}^{\mathrm{CI}}=\operatorname{sgn}\left(\operatorname{Re}\left[\boldsymbol{y}^{\mathrm{CI}}\right]\right)
$$

The classical MMSE and ZF methods are decoded using

$$
\begin{aligned}
\widehat{\boldsymbol{b}}^{\mathrm{MMSE}} & =\operatorname{sgn}\left\{\operatorname{Re}\left[\left(\sigma_{n}^{2} \boldsymbol{I}+\boldsymbol{H}^{\dagger} \boldsymbol{H}\right)^{-1} \boldsymbol{H}^{\dagger}(\boldsymbol{H} \boldsymbol{b}+\boldsymbol{n})\right]\right\} \\
\widehat{\boldsymbol{b}}^{\mathrm{ZF}} & =\operatorname{sgn}\left\{\operatorname{Re}\left[\left(\boldsymbol{H}^{\dagger} \boldsymbol{H}\right)^{-1} \boldsymbol{H}^{\dagger}(\boldsymbol{H} \boldsymbol{b}+\boldsymbol{n})\right]\right\}
\end{aligned}
$$

The ZF method requires that $n_{\mathrm{T}} \leq n_{\mathrm{R}}$.

Fig. 4 shows our simulated symbol error probabilities against SNR for all four MIMO methods using the $(2,2)$ and $(4,4)$ antenna configurations for the i.i.d channels. We see that for both configurations, the MMSE method outperforms the other methods, given equal signal power allocation among the parallel channels for the SVD and CI methods and among the transmitting antennas for the MMSE and ZF methods. The other three methods have

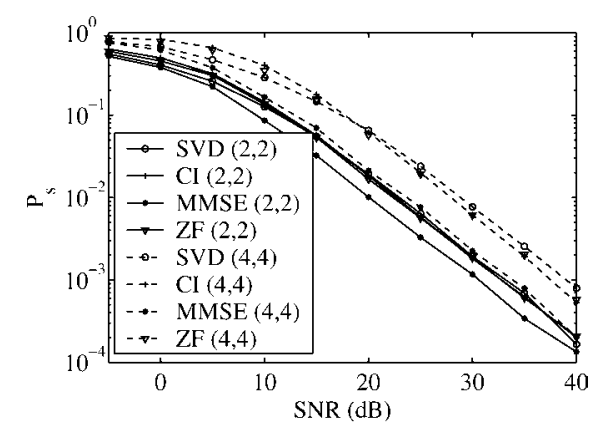

Fig. 4 Simulated symbol error probabilities for i.i.d. Rayleigh case 
very similar performances. The MMSE method utilises both the channel and noise statistics, whereas the other three methods only use the channel statistics. Note that the error rate performance of MIMO-MMSE systems has been studied in [19]. Unfortunately, with the symbol error probability dominated by the worst eigen-channels, the considered equal-power version of the SVD method does not exploit the better eigenchannels. The advantage of the SVD method lies in the ability to 'water-fill' the eigenchannels with different-sized signal constellations, increasing the capacity of the MIMO system for a given symbol error probability. Finally, note that the diversity gains of the four methods are the same.

Fig. 5 shows our calculated and simulated symbol error probabilities against SNR for $(2,2),(2,4),(4,2)$ and $(4,4)$ MIMO systems for the semi correlated case. We have calculated $P_{\mathrm{s}}$ for a $\lambda / 2$-spaced transmitter or receiver (depending on which has fewer antennas) with high spatial correlation $\Theta_{c}$ defined for the generalised International Telecommunication Union (ITU) pedestrian environment A for a macrocell with Laplacian power azimuth spectrum and a $5^{\circ}$ angle spread [20]. Again, our calculations line up very well with our simulations that are based on 500000 Monte Carlo runs. Note that the large correlation produces a larger eigenvalue spread with smaller $\lambda_{\min }$ than the i.i.d. Rayleigh case. Since $\lambda_{\min }$ dominates $P_{\mathrm{s}}$, the semi-correlated case performances are inferior to those of the i.i.d. case.

For this semi-correlated Rayleigh case, we compare the SVD method with the CI, MMSE and ZF methods. Fig. 6 shows $P_{\mathrm{s}}$ against SNR for all four MIMO methods using $(2,2)$ and $(4,4)$ antenna configurations. Again, because of the lack of 'water-filling' in the SVD method and the intelligent use of the noise statistics, the MMSE method outperforms the other methods with the other three methods having very similar performances.

Fig. 7 shows our calculated and simulated $P_{\mathrm{s}}$ against SNR for $(2,2),(2,4),(4,2)$ and $(4,4)$ MIMO systems for a Ricean channel. We have calculated the symbol error probabilities for the rank one Ricean channel with fixed angles $\theta_{\mathrm{t}}=20^{\circ}$ and $\theta_{\mathrm{r}}=35^{\circ}, d=4$, and $K=10 \mathrm{~dB}$. Here the values of these parameters were selected to verify our analysis rather than to model a particular array environment. An antenna spacing of $d=4$ wavelengths would typically correspond to a base station. Again, our calculations line up very well with our simulations which are based on 500000 Monte Carlo runs. The simulated values and theoretical curves differ slightly for the $(4,4)$ case at large SNRs because of the instability of the numerical integral (23). Relative to the i.i.d. Rayleigh case, the line of sight component in the Ricean case produces a larger eigenvalue spread with smaller $\lambda_{\min }$, particularly for large $m$. Since

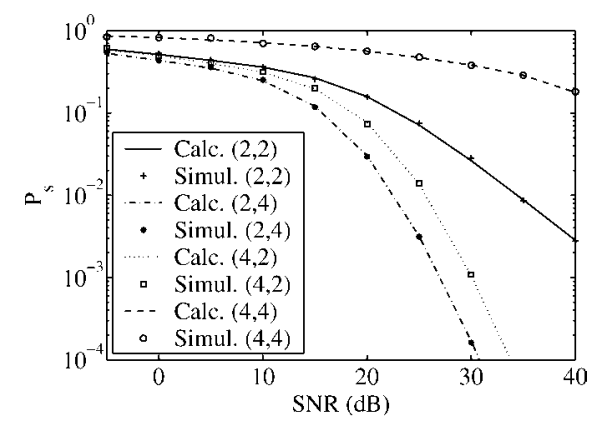

Fig. 5 Symbol error probabilities for semi-correlated Rayleigh case

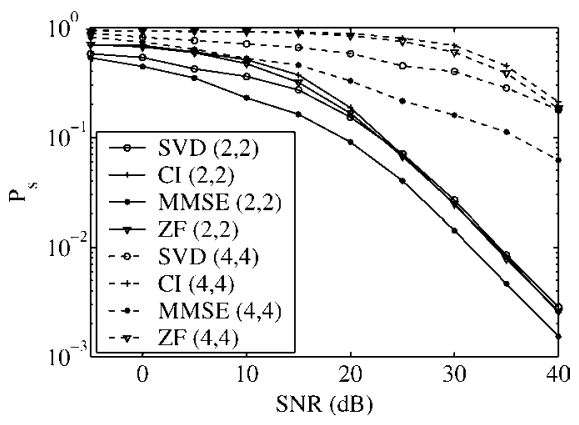

Fig. 6 Simulated symbol error probabilities for semi-correlated Rayleigh case

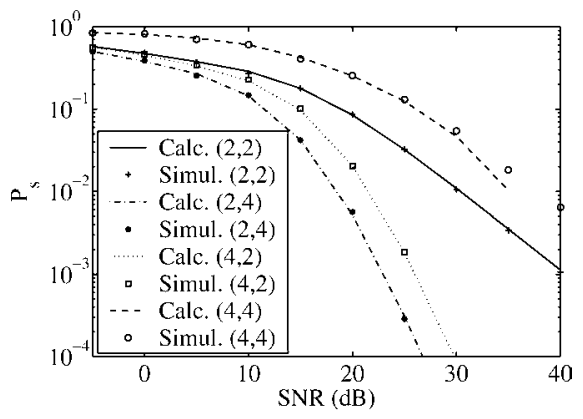

Fig. 7 Symbol error probabilities for rank 1 Ricean case

$\lambda_{\text {min }}$ dominates $P_{\mathrm{s}}$, the Ricean case performances are inferior to those of the i.i.d. Rayleigh case.

\subsection{Single-user case with inter-cell interference}

In this section, we consider the impact of other cell interference. We assume that the MIMO system uses a TDMA protocol and at a particular time only one user is active in the desired cell, but there are also other users active in the surrounding cells. Hence, there is no intra-cell interference but there is inter-cell interference.

Fig. 8 shows the error free rate, $R_{\mathrm{T}}$, for the adaptive MIMO SVD scheme, with a target BER of $10^{-1}$. Results are shown for a range of fixed SINR values from -5 to $15 \mathrm{~dB}$ for $(1,1),(2,2),(4,2)$ and $(4,4)$ schemes. The lines indicate analytical calculations obtained from (34). The points represent simulation results. Note the slight difference between the simulations and the analysis because of the use of the BER approximation in (28), which leads to the analytical calculations being marginally below the simulated results. Fig. 8 is a useful verification of the analysis method and clearly shows the massive

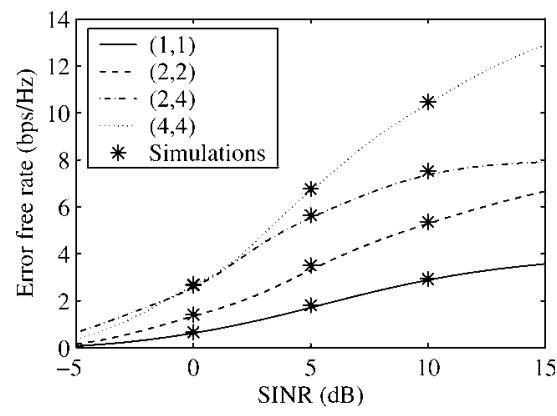

Fig. 8 Error free rate against $\operatorname{SINR}$ for $(1,1),(2,2),(2,4)$ and $(4,4)$ systems, with a target BER of $10^{-1}$ 


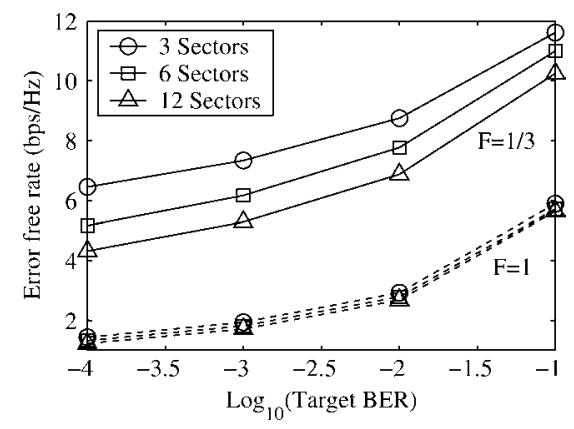

Fig. 9 Error free rate against target BER for all six cellular scenarios for a $(4,4)$ system

drop in $R$ as the SINR is reduced, especially for larger systems.

Fig. 9 is a central result and shows the trade off between BER and $R$ for a $(4,4)$ system. For target BERs down at $10^{-4}$, the values of $R$ are generally less than half the values at a target of $10^{-1}$. Clearly, with the cellular SINR distributions considered, the overall error free rate is far from the maximum of 16 (number of transmit antenna times the bits/symbol for 16 QAM). At acceptable BER values, even the best case only gives values around $6-7$, and the worst case is between 1 and 2 .

Next we consider slight variations in the adaptive SVD scheme. In particular, we remove the 16-QAM option or the suspended transmission mode. If the suspended transmission mode is removed, then transmission always occurs, even on the worst eigenmode. Fig. 10 shows a comparison of the performance of the standard scheme with these 2 variations. The effect of removing the 16-QAM option is very large, and the values of $R$ drop markedly. Clearly, it is very important to be able to use the highest modulation schemes for periods of large SINR. Removing the suspended transmission mode increases $R$, but the change is not so dramatic and brings with it increased BER.

The results to date show that the adaptive scheme performs far below the maximum possible. Hence, it is important to compare the adaptive scheme with other detection methods. Therefore we also consider MIMO-MMSE combiners of the form (39) operating with fixed BPSK or 4 -QAM modulations. All system sizes from $(1,1)$ to $(4,4)$ were simulated, and for each system both $R$ and the average BER, averaged over all SINR values, were simulated. In addition, the adaptive SVD was considered over a range of target BERs. At each target BER, the average BER was evaluated by simulation and $R$ was obtained from (35). Results are shown in Fig. 11. In comparison to the fixed transmission scheme, the adaptive scheme performs far better. Its ability to use higher-order modulations

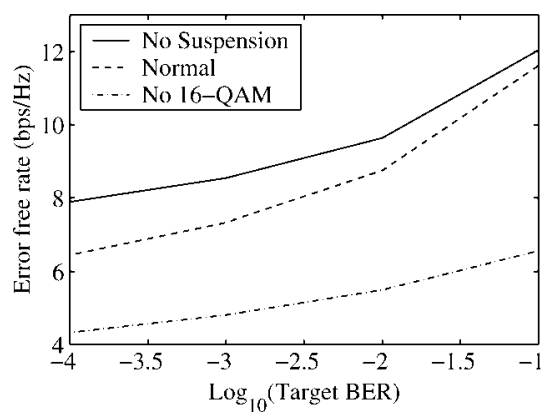

Fig. 10 Error free rate against target BER for cellular scenario $R 3 S 3$, with three versions of adaptive transmission for a $(4,4)$ system

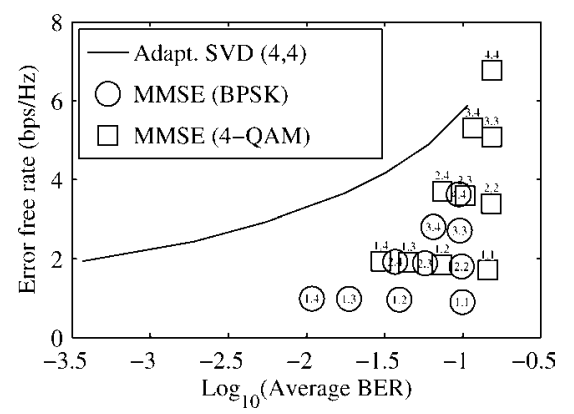

Fig. 11 Error free rate against average BER for SVD adaptive transmission and MIMO-MMSE linear combiners, for cellular scenario R1S3

at high SINR and reduce the constellation size or suspend transmission during very low SINR periods allows it to maintain reasonable values of $R$ at average BER values, well below those offered by the MIMO-MMSE receivers.

Fig. 12 illustrates the behavior of the probability that transmission is completely suspended on all eigenchannels, $P_{\mathrm{S}}$. This can be obtained from $P_{\mathrm{S}}=P\left(\lambda_{\max }<q_{1}\right)$, and these can be computed analytically by integrating (7). Fig. 12 shows how $P_{\mathrm{S}}$ drops with SINR for various system sizes using a target BER of $10^{-3}$. The $(1,1)$ system requires large SINR values around $20 \mathrm{~dB}$ before $P_{\mathrm{S}}$ approaches zero. The $(4,4)$ system is best, but still requires around $5 \mathrm{~dB}$, and inspection of the $\mathrm{CDFs}$ for $F=1$ shows that this SINR is available with a probability less than 0.5 .

In Figs. 13 and 14, we consider the comparative performance of diversity against MIMO and adaptive against fixed modulations. Fig. 13 shows the error free rates achieved by various diversity schemes with the $(4,4)$ system also shown for comparison. All systems use the standard adaptive approach, employing 16-QAM, 4-QAM, BPSK or transmission suspension, depending on the channel. At all

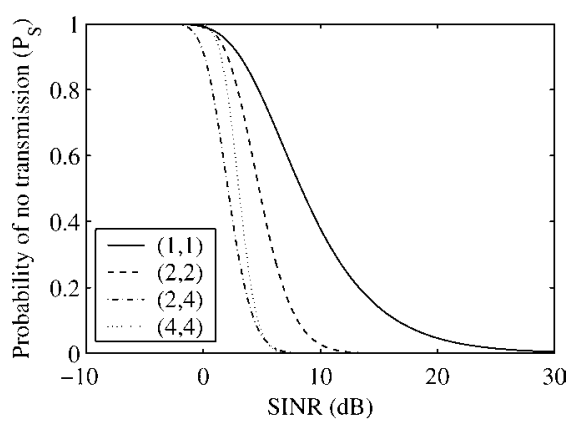

Fig. 12 Probability of no transmission against SINR for $(1,1)$, $(2,2),(2,4)$ and $(4,4)$ systems, with a target BER of $10^{-3}$

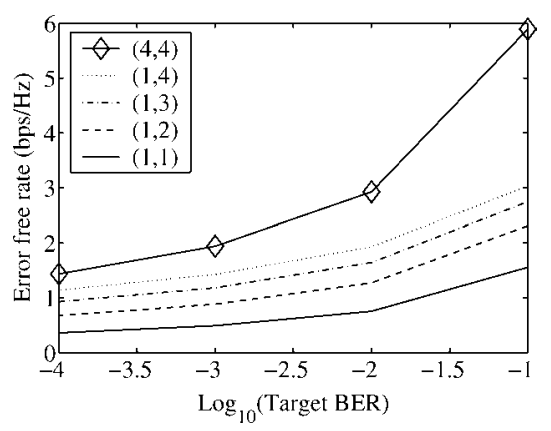

Fig. 13 Error free rate against target BER: effect of $R X$ diversity on SVD adaptive transmission for cellular scenario RIS3 


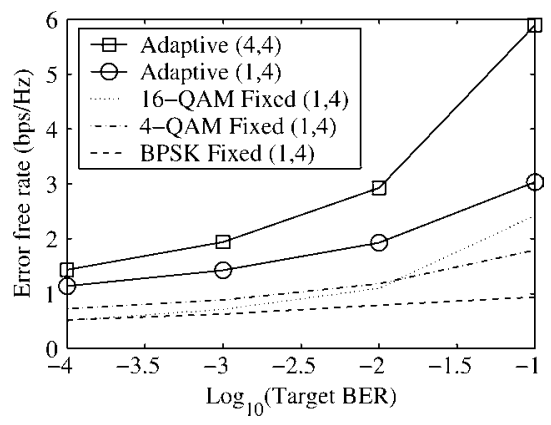

Fig. 14 Error free rate against target BER: adaptive against fixed SVD modulation schemes for cellular scenario R1S3

target BERs increased diversity yields improved rates, and at low target BERs the $(1,4)$ scheme is only marginally worse than the $(4,4)$ system. Fig. 14 shows the impact of adaptive modulation. The bottom 3 curves are for a scheme where transmission is either suspended or a fixed 16-QAM, 4-QAM or BPSK modulation is employed. In the legend, such a scheme is labelled as 'Fixed'. The standard adaptive scheme for $(1,4)$ and $(4,4)$ systems are also shown for comparison. At low target BERs the adaptive $(1,4)$ scheme offers more than a $60 \%$ improvement over the fixed modulations. The improvement over the $(1,4)$ diversity approach offered by the $(4,4)$ system is less than $30 \%$.

\section{Conclusion}

In this paper, we have derived exact symbol error probabilities for SVD-based MIMO systems and have compared their simulated performance with CI, MMSE and ZF receivers. These error probabilities for the SVD-based MIMO systems provide new insights. Although the capacity of MIMO systems increases with the number of antennas, the error performance degrades. This suggests that capacity increases and error performance need to be carefully considered. Finally, to take full advantage of the CSI information at the transmitter, the analysis of the SVD method should be extended to higher-order signal constellations (M-PSK and M-QAM) and adaptive 'water-filling' schemes.

We have also derived and verified the error free rate of an adaptive MIMO SVD system. This performance metric is useful in comparing both fixed and adaptive transmission schemes with varying constellation sizes. We have shown the effect of a cellular environment on performance, specifically the effects of cellular layout and the variance of the SINR distribution. We have presented key results concerning the effect of adaptive modulation and the performance gap between diversity methods and MIMO. In particular, we have found that the improvement offered by the adaptive SVD approach is at the expense of suspending transmission, and these periods of no communication occur with high probability at SINR values below $5 \mathrm{~dB}$. For the cellular layouts considered, these SINR values are extremely common. Finally, we have demonstrated that the improvement of the $(4,4)$ adaptive SVD scheme over the $(1,4)$ diversity scheme is relatively small, considering the complexity increase and feedback requirements. We conclude that in the cellular environments considered, relative to a SISO system, the majority of the performance gain due to the $(4,4)$ system can be obtained via an adaptive diversity system.

\section{Acknowledgment}

This work was partially supported by the Foundation for Research, Science and Technology of New Zealand and Telecom New Zealand Limited. Parts of this work were presented at the IEEE ICC Conference, Seoul, South Korea in 2005 and at the AusCTW Workshop, Perth, Australia in 2006.

\section{References}

1 Telatar, I.E.: 'Capacity of multi-antenna Gaussian channels', Eur. Trans. Telecommun. Relat. Technol., 1999, 10, pp. 585-595

2 Foschini, G.J., and Gans, M.J.: 'On limits of wireless communication in a fading environment when using multiple antennas', Wirel. Pers. Commun., 1998, 6, (3), pp. 311-335

3 Huang, H., and Valenzuela, R.: 'Fundamental simulated performance of downlink fixed wireless cellular networks with multiple antennas'. Proc. IEEE Int'l. Symp. Personal and Mobile Radio Communications, Berlin, Germany, 11-14 September 2005

4 Garth, L.M., Smith, P.J., and Shafi, M.: 'Exact symbol error probabilities for SVD transmission of BPSK data over fading channels'. Proc. 2005 IEEE Int. Conf. Communications, Seoul, Korea, 16-20 May, 2005, pp. 2271-2276

5 Zanella, A., Chiani, M., and Win, M.Z.: 'Performance of MIMO MRC in correlated Rayleigh fading environments'. Proc. 61st IEEE Vehicular Technology Conf., VTC Spring 2005, Stockholm, Sweden, 30 May - 1 June 2005, pp. 1633-1637

6 Garcia-Ordonez, L., Palomar, D.P., Pages-Zamora, A., and Fonollosa, J.R.: 'Analytical BER performance in spatial multiplexing MIMO systems'. Proc. 6th IEEE Workshop on Signal Processing Advances in Wireless Communications, New York, 5-8 June 2005, pp. $460-464$

7 Raleigh, G.G., and Cioffi, J.M.: 'Spatio-temporal coding for wireless communication', IEEE Trans. Commun., 1998, 46, (3), pp. 357-366

8 Lebrun, G., Ying, T., and Faulkner, M.: 'MIMO transmission over a time-varyingchannel using SVD'. Proc. 2002 IEEE Global Telecommunications Conf., Taipei, Taiwan, 17-21 November 2002, pp. $414-418$

9 Grant, M.: 'Draft report on radio aspects for the terrestrial component of IMT-2000 and systems beyond IMT-2000', ITU Document 8F/ TEMP/216 (Rev. 3), Attachment 6.9, 30 September, 2005

10 James, A.T.: 'Distributions of matrix variates and latent roots derived fromnormal samples', Ann. Math. Stat., 1964, 35, (1), pp. 475-501

11 Chiani, M., Win, M.Z., and Zanella, A.: 'On the capacity of spatially correlated MIMO Rayleigh-fading channels', IEEE Trans. Inform. Theory, 2003, 49, (10), pp. 2363-2371

12 Proakis, J.G.: 'Digital communications' (McGraw-Hill, New York, 2001, 4th edn.)

13 Smith, P.J., Garth, L.M., and Loyka, S.: 'Exact capacity distributions for MIMO systems with small numbers of antennas', IEEE Commun. Lett., 2003, 7, (10), pp. 481-483

14 Farrokhi, F.R., Foschini, G.J., Lozano, A., and Valenzuela, R.: 'Link-optimal space-time processing with multiple transmit and receive antennas', IEEE Commun. Lett., 2001, 5, (3), pp. 85-87

15 Smith, P.J., and Garth, L.M.: 'Exact capacity distributions for dual MIMO systems in Ricean fading', IEEE Commun. Lett., 2004, 8, (1), pp. 18-20

16 Smith, P.J., and Garth, L.M.: 'Distribution and characteristic functions of correlated complex Wishart matrices', submitted to J. Multivariate Anal., 2004

17 Simon, M.K., and Alouini, M.-S.: 'Digital communication over fading channels: a unified approach to performance analysis' (John Wiley \& Sons, New York, 2000)

18 Haustein, T., von Helmolt, C., Jorswieck, E., Jungnickel, V., and Pohl, V.: 'Performance of MIMO systems with channel inversion'. Proc. 55th IEEE Vehicular Technology Conf. VTC Spring 2002, Birmingham, Alabama, 6-9 May 2002, pp. 35-39

19 Zanella, A., Chiani, M., and Win, M.Z.: 'MMSE reception and successive interference cancellation for MIMO systems with high spectral efficiency', IEEE Trans. Wirel. Commun., 2005, 4, (3), pp. $1244-1253$

20 3GPP: 'Initial channel models for MIMO', TSGR1\#21(01)0902, Turin, Italy, 27-31 August, 2001 